\title{
Blood pressure variability parameters during ibuprofen induced closure of the ductus arteriosus
}

\author{
Frank Bennis ${ }^{A}, \mathrm{~B}$, Peter AndriessenC, Carola van PulD, Boris W. Kramer ${ }^{\mathrm{B}, \mathrm{E}}$, Tammo Delhaas ${ }^{\mathrm{A}, \mathrm{F}}$ \\ Department of Biomedical Engineering, Maastricht, The Netherlands \\ MHeNS School for Mental Health and Neuroscience, Maastricht University, Maastricht, The Netherlands \\ Department of Pediatrics, Máxima Medical Centre, Veldhoven, The Netherlands \\ Department of Clinical Physics, Máxima Medical Centre, Veldhoven, The Netherlands \\ Department of Pediatrics, Maastricht University, Maastricht, The Netherlands \\ School for Cardiovascular Diseases, Maastricht University, Maastricht, The Netherlands
}

\section{Introduction}

Hemodynamically significant patent ductus arteriosus (HS-PDA) in preterm infants are diagnosed with echocardiography and treated with a 3-day ibuprofen treatment course. Though closure is confirmed by a second echo, status of ductal closure during treatment is unknown.

\section{Hypothesis}

Changes in blood pressure (BP) variability can indicate ductal closure during medical treatment.

\section{Results}

Ductal closure during treatment was associated with a significant increase in low frequency power relative to baseline as well as a significant decrease in beat-to-beat changes in BP. Furthermore, the systolic standard deviation increased during ductal closure.

\section{Conclusion}

BP variability has clinical potential to monitor ductal closure during medical treatment.

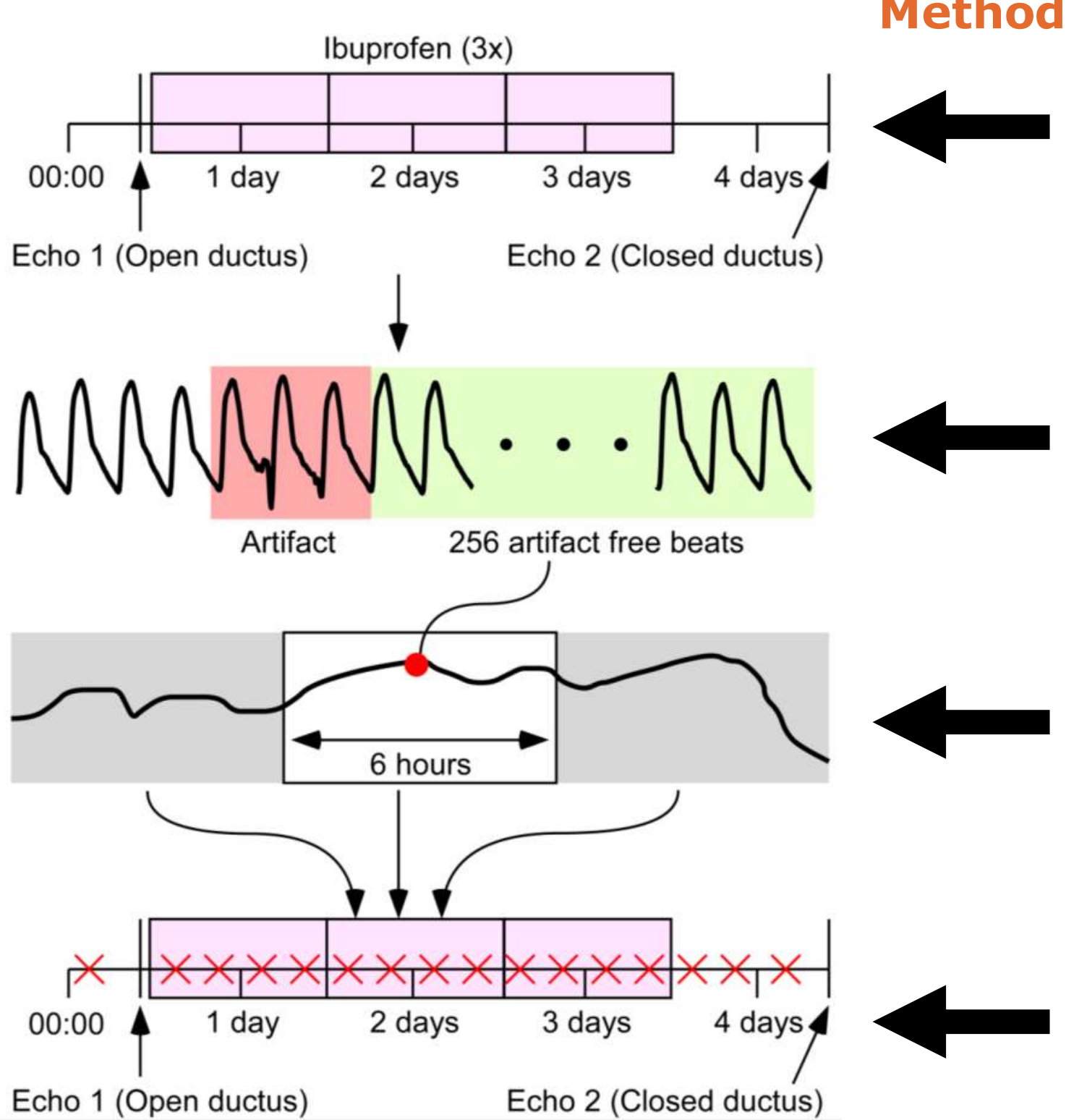

Figure 1. Overview of methods

\section{Study group:}

- Ten preterm infants $(25+4$ to $27+4$ weeks gestation, $520-1080 \mathrm{~g}$ birth weight) with HS-PDA that closed after 3-day ibuprofen. Datatype:

Arterial BP waveforms from start day echo 1 until echo 2.

\section{Preprocessing:}

Detection of systolic BP and diastolic BP peak values in $\mathrm{mmHg}$

Automated beat-to-beat artifact removal (5.8 $2.2 \%$ removed).

- Artifact-free 256-beat window selection (19.3 \pm 5.4 segments/hr).

Parameter calculation for diastolic and systolic values: -Time domain: standard deviation (SD), mean, coefficient of variation $(\mathrm{CV})$ and root-mean-square of successive differences (RMSSD).

-Frequency domain: power low frequency (LF, $0.04 \mathrm{~Hz}$ to 0.2 $\mathrm{Hz}$ ), power high frequency (HF, $0.2 \mathrm{~Hz}$ to $1 / 2$ heart rate $\mathrm{Hz}$ ) and total power $(0.04 \mathrm{~Hz}$ to $1 / 2$ heart rate $\mathrm{Hz})$.

\section{Baseline comparison}

Median parameter values over all six-hour windows were calculated and visualized (Figure 2).

Change in median from 00:00 to 06:00 before first echo (open ductus, baseline) relative to same period before second echo (closed ductus) (Figure 3).

\section{Results}

Change in diastolic low frequency power over time

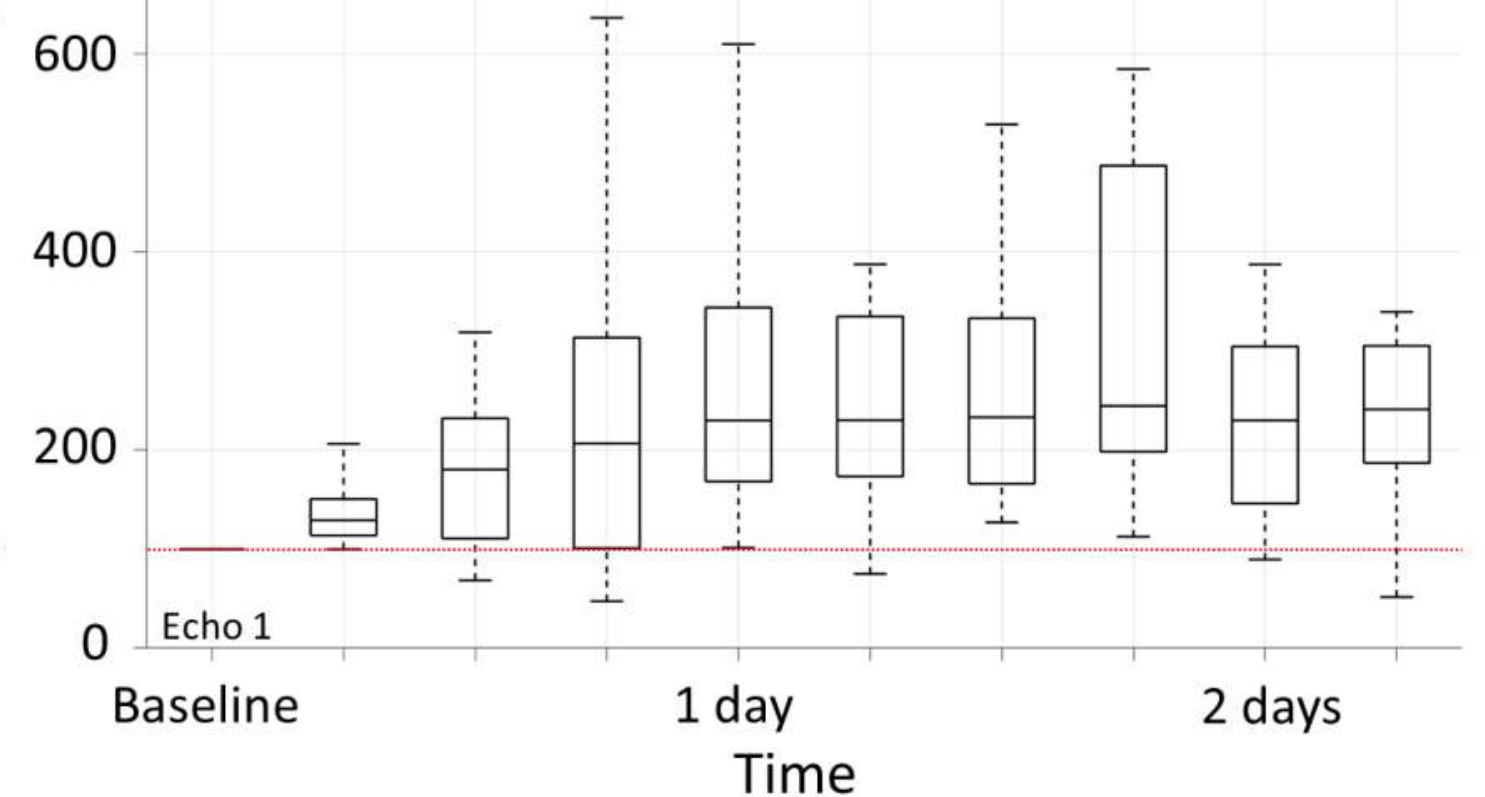

Figure 2. Progress of diastolic LF power over time. Each boxplot indicates a 6 hour window. An increase in power can be seen after echo 1, possibly indicating rapid closure. All boxplots show significant differences with respect to the baseline.

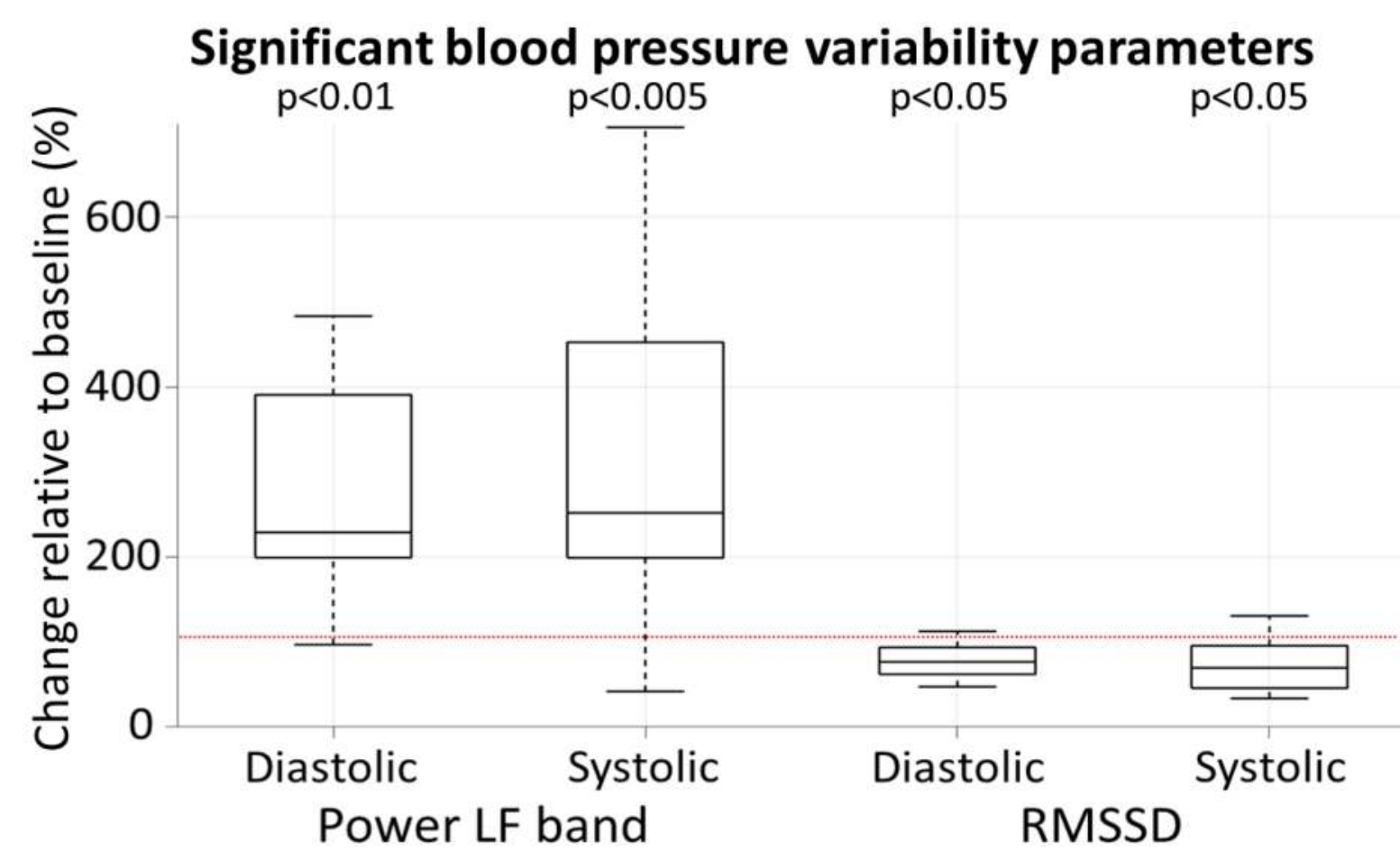

Figure 3. Boxplot of the change relative to baseline (red dotted line) for the power in the low frequency domain and the RMSSD (all significant). The $x$-axis shows the parameters, the $y$-axis the percentage change. 